\title{
Perspectives on Mucus Properties and Formation-Lessons from the Biochemical World
}

\author{
Daniel Ambort, Malin E.V. Johansson, Jenny K. Gustafsson, Anna Ermund, \\ and Gunnar C. Hansson
}

Department of Medical Biochemistry, University of Gothenburg, 40530 Gothenburg, Sweden

Correspondence: gunnar.hansson@medkem.gu.se

Our model of the MUC2 mucin shows a well-organized netlike gel that is cross-linked by six different covalent and noncovalent bonds. When the MUC2 mucin is packed in the mucin granule it is organized by an amino-terminal concatenated ring platform formed at high calcium and low $\mathrm{pH}$. This packing allows an ordered release and a normal mucin expansion when calcium is removed and $\mathrm{pH}$ increased by bicarbonate. This process is defective in the absence of cystic fibrosis transmembrane conductance regulator (CFTR)-dependent bicarbonate transport. The expanded secreted mucin is suggested to be self-organizing by properties inherited in the MUC2 mucin and by proteolytic processes.

M

ost of the organs affected in cystic fibrosis (CF) patients, except the sweat glands, can be attributed to the $\mathrm{CF}$ viscous mucus phenotype. Tubular organs, such as exocrine pancreas and epididymis, are often clogged by mucus already at birth. The small intestine can also be obstructed by the mucus-rich meconium to give meconium ileus at birth. CF adults often also experience intestinal pain and sometimes DIOS (distal ileum obstruction syndrome). However, most commonly and seriously, the slow and stagnant mucus of the lungs give persistent bacterial lung infections.

What is mucus then? Mucus is the gel-like material found on all mucosal surfaces. Its major component is one or several gel-forming mucins, discussed by Kreda et al. (2012). Mucus, depending on the organ, contains a num- ber of specific components in addition to the mucins, such as, for example, exfoliated cells and other trapped materials. The function of mucus is to protect the underlying epithelium and to act like glue, which can trap and transport away debris and microorganisms found in the mucus gel. The CF pathologies can, in a simplified way, be understood as mucus that is trapped and stickier than normal.

Understanding mucus requires an understanding of mucins, which has, however, proven to be difficult as is reflected by this and Verdugo (2012). Verdugo (2012) and this article together give two almost completely contradictory models of how mucins are organized, largely because each group of investigators takes a different scientific starting point. Verdugo views mucus from "above" and explains its properties in

Editors: John R. Riordan, Richard C. Boucher, and Paul M. Quinton

Additional Perspectives on Cystic Fibrosis available at www.perspectivesinmedicine.org

Copyright (C) 2012 Cold Spring Harbor Laboratory Press; all rights reserved; doi: 10.1101/cshperspect.a014159

Cite this article as Cold Spring Harb Perspect Med 2012;2:a014159 
D. Ambort et al.

terms of biophysical and macromolecular approaches generated in the polymer science of large synthetic polymers. The view in the present work, however, is based on recently acquired knowledge of mucin biochemistry and the characterizations of molecular details of gelforming mucins. This views mucus from "below" and tries to assemble more detailed knowledge into models of mucus and mucus function. At first glance, these two models may seem totally incongruent. However, this is probably not the case because different mucus gels, formed by different mucins, show different organization and function. Before describing the biochemical model of mucins, one major feature must be discussed, the glycans.

\section{THE DENSELY O-GLYCOSYLATED MUCIN DOMAINS}

A high content of glycans, especially the $O$ glycans (or as they are often incorrectly referred to, the mucin glycans) is typical of mucins. These glycans are densely clustered in the so-called mucin domains of the protein core to extend and make the mucin domains rodlike (Andersch-Björkman et al. 2007; Lang et al. 2007; Holmen Larsson et al. 2009). A bottle brush with its metal core in the center as the mucin protein backbone and the glycans forming the bristles are often used to explain this. The protein core is rich in the amino acids serine and threonine that anchor the $O$ glycans. These amino acids are interspersed with prolines giving the peptide a random coil structure that allows the peptidyl-GalNAc transferases of the Golgi apparatus to attach $N$-acetylgalactosamine as the first $O$-linked sugar. The amino acid sequences of these domains have been poorly conserved during evolution, but can be identified as PTS sequences in the genome as long stretches of nucleotides that encode high frequencies of the three amino acids: proline, threonine, and serine (PTS) (Lang et al. 2004, 2007). These PTS sequences often contain more or less perfectly repeated sequences, but this is not always the case. These sequences are often called VNTR for variable number of tandem repeats, but this name is misleading as there are a number of mu- cins with heavily degenerated repeats and no repeated sequences can be identified. Nonetheless, long stretches of $>100$ amino acids with a high frequency of the proline, threonine, and serine amino acids are characteristic of these domains.

The highly glycosylated mucin domains have, for mucins, the important biophysical property of binding water. This and their polymeric nature generate the gel-like properties typical for mucins. The numerous hydroxyl groups of the individual monosaccharides account for most of the high water-binding capacity. The presence of negatively charged groups in the form of carboxyl groups on the sialic acids and sulfate groups contributes further to waterand ion-binding capacities. As the glycosylation is encoded by specific transferases, the glycans on one and the same mucin protein core can vary enormously in different organs and even more so among the same organ in different species. These differences are, for example, determined by blood-group-type glycan epitopes (i.e., $\mathrm{ABH}$, Lewis, etc., histo-blood-group systems). Such polymorphisms are beneficial for the host in constant battle against pathogenic organisms (Gagneux and Varki 1999). Although still debated, it is less likely that specific mucin glycans contribute very much to differences in mucus properties.

\section{THE BIOCHEMICAL PERSPECTIVE ON MUCINS}

Our view of mucins and how they form mucus starts with the MUC2 mucin (Johansson et al. 2011). This is the major mucin in the small and large intestine and as such can be expected to be exposed to the harshest conditions of all mucins (Gum et al. 1994). This will probably place the MUC2 mucin at one extreme and as such be one reason for its properties as compared with the linear polymer model traditionally given to mucins as discussed by Verdugo (2012). A major conclusion from our work on the MUC2 mucin is that mucus gels are very well-organized structures, much more than considered previously. This organization is based on the structure of its major building block, the MUC2 mucin monomer (Fig. 1). 


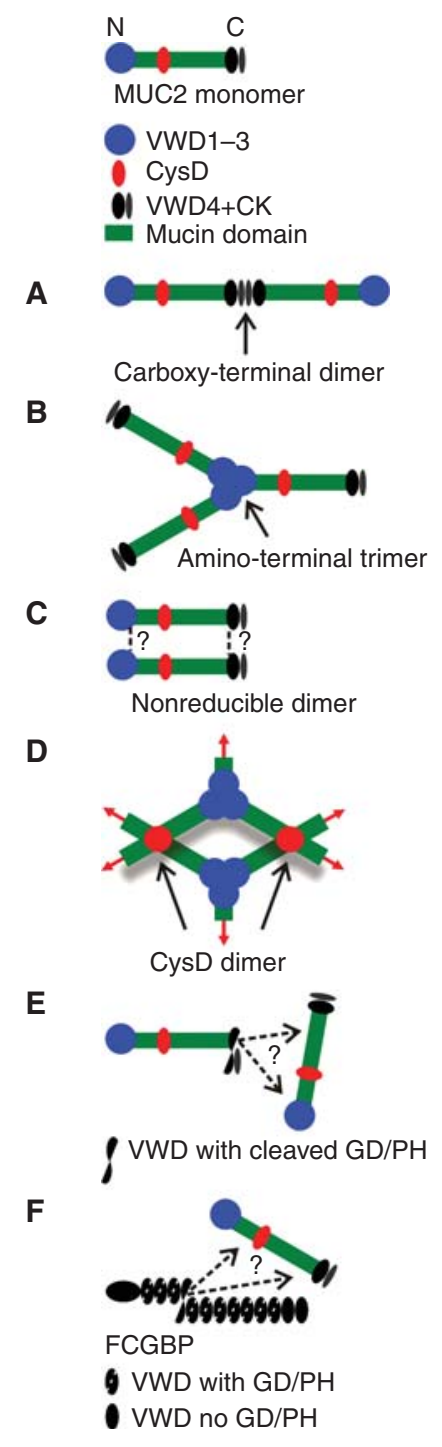

Figure 1. A schematic view of the MUC2 mucin (top). The amino-terminal von Willebrand D1, D2, and D3 (VWD1-3) domains comprising the first 1200 amino acids are given as a blue dot. The two mucin domains are green. The CysD domains are red (only one of the two is shown). The carboxy-terminal 980 amino acids, including the von Willebrand D4 (VWD4) and the cysteine-knot domains are given in black. (A) Disulfide-bonded dimeric carboxy terminus as formed in the endoplasmic reticulum. $(B)$ Disulfide-bonded trimeric amino terminus as formed in the secretory vesicles. $(C)$ Nonreducible covalent bond formed in the late secretory pathway. $(D)$ CysD domains form noncovalent dimeric complexes. (E) The autocatalytic cleavage at the GD/PH bond (between aspartate and proline in the sequence
The MUC2 mucin builds the structure of the two-layered mucus of colon (Johansson et al. 2008). The inner mucus layer that is 50 - to $100-\mu \mathrm{m}$ thick (mouse) is anchored to the epithelium and impenetrable to bacteria. Thus, this layer keeps all the bacteria at a distance from the underlying epithelial cells. This inner layer is converted to an outer mucus layer that is less dense and forms the habitat of the commensal bacteria (Johansson et al. 2011). The inner layer has a stratified appearance and seems well organized. The organization of the MUC2 mucin in the mucus must be spontaneous owing to builtin properties and interactions.

As discussed by Verdugo (2012), the view of mucins as long linear polymers that give gel-like properties by entanglement has dominated the mucin field for decades. In contrast, we argue that the mucus gel is formed by a heavily crosslinked network with intrinsic biochemical interactions and properties. Today we know of six different types of covalent and noncovalent interactions within the MUC2 mucin that are all, except one, incompatible with a model of entangled linear polymers as a structural basis for mucins.

The MUC2 monomer is made of $5174 \mathrm{ami}-$ no acids organized in an amino terminus with three complete and one partial von Willebrand D domains (VWD), two PTS sequences that give two mucin domains, and a carboxy-terminal end of 840 amino acids with one VWD and a cysteine-knot (CK) domain (Fig. 1, top) (Gum et al. 1994). The MUC2 mucin also contains two CysD domains in the central PTS-containing region (only the second one between the two mucin domains is shown in Fig. 1).

After folding in the endoplasmic reticulum, the primary translational MUC2 product is directly dimerized via its carboxy-terminal end

Figure 1. (Continued) glycine-aspartate-proline-histidine) in the carboxy-terminal von Willebrand D4 domain generates a new carboxy terminus with an internal anhydride that can cross-link MUC2. $(F)$ FCGBP has 11 von Willebrand D domains (VWD) with a cleavage GD/PH site and two without. As for MUC2, the GD/PH bond is cleaved and will crosslink, for example, MUC2. 
D. Ambort et al.

CK (Fig. 1A). Disulfide bonds between the CK domains form this dimer as in other dimeric proteins with CK domains (Asker et al. 1995, 1998; Lidell et al. 2003b). The dimers are then transported into the Golgi apparatus to be $\mathrm{O}$ glycosylated. The mass of the dimer increases about fivefold owing to the glycans, to $\sim 5 \mathrm{MDa}$.

In the trans-Golgi network, the mucin dimers are sorted to the regulated secretory pathway and the MUC2 amino termini form disulfide-bonded trimers (Fig. 1B) (Godl et al. 2002). The trimerization takes place in the VWD3 domain, a part of the molecule that is very dense and has the appearance of a trefoil when studied by molecular electron microscopy. There have been some discussions on the trimeric nature of the MUC2 amino termini, but these have been based solely on migration on SDS-polyacrylamide gels (Heazlewood et al. 2008). The reason for this discussion is that the nonreduced trimer is just a bit larger than two times the size of the reduced monomer. However, this is in fact proof of the nonreduced MUC2 being trimeric as the nonreduced MUC2 monomer is compact and migrates faster than the reduced form. Also, the porcine submaxillary mucin (PSM) forms amino-terminal trimers (Perez-Vilar and Hill 1998). Unfortunately, we do not have any details of the other human mucins, as these have not been expressed as truncated forms that would allow any final conclusions about their dimeric or trimeric amino-terminal nature. However, recent studies of the MUC6 amino termini also suggest a trimeric organization (Leir and Harris 2011). It is possible that some mucins still have amino-terminal dimeric forms as first assumed (Gum et al. 1994), parallel to the von Willebrand factor that is dimeric, and thus form linear polymers (Sadler 2009). Some biophysical and biochemical data may suggest that the MUC5B mucin and perhaps the MUC5AC are dimeric (Sheehan et al. 2004; Thornton et al. 2008).

During the later stages of the secretory pathway, probably after sorting to the regulated pathway and at the time of trimerization, the MUC2 mucin becomes insoluble in normal buffers (Axelsson et al. 1998). This occurs at the same time the nonreducible bond is formed as indicated by the appearance of nonreducible dimers after reduction of the disulfide bonds analyzed by gel electrophoresis. The same type of bond was first observed in mature, secreted mucus from the intestine, where the MUC2 mucin is also insoluble in chaotropic salts like guanidinium chloride (Carlstedt et al. 1993; Herrmann et al. 1999). The nature and localization of the nonreducible covalent bond remains a mystery (Fig. 1C).

The MUC2, MUC5AC, and MUC5B mucins all contain two or more CysD domains. This domain is 100 amino acids long and contains 10 conserved Cys residues. After intensive studies on the second CysD of the MUC2 mucin we have concluded that these domains form noncovalent dimers that are held together by strong hydrophobic forces (Fig. 1D) (Ambort et al. 2011). That no disulfide bonds are involved in forming the dimers became evident from the observation that all five disulfide bonds could be attributed to intramolecular disulfide bonds. It was previously suggested that the CysD domains of MUC5AC do not interact (Perez-Vilar et al. 2004), but our present and unpublished results strongly indicate that like the MUC2 CysD domains, MUC5AC CysD domains also form noncovalent dimers.

During our studies of the MUC2 mucin, we have also found two additional types of MUC2 cross-links. An autocatalytic cleavage of the VWD4 domain at the GD/PH amino acid sequence was found to be enhanced at a $\mathrm{pH}$ close to 6 (Fig. 1E) (Lidell et al. 2003a). This type of cleavage also occurs in the VWD4 of the MUC5AC mucin (Lidell and Hansson 2006). The new carboxy-terminal aspartate forms an internal anhydride between its new carboxyterminal carboxyl group and its side chain. Such anhydrides are very reactive, both with primary amines and hydroxyl groups. As such groups are numerous on mucins, especially hydroxyl groups, it is very likely that covalent bonds will be formed in mucus and thereby stabilize the mucin network just as fibrin is stabilized for mechanical strength. It should also be pointed out that the MUC2 and MUC5AC mucin polymers are still held together over the $\mathrm{GD} / \mathrm{PH}$ cleavage by one or several disulfide bonds that bridge this sequence. 
Recently, we showed that another protein, FCGBP, is covalently bound to MUC2 mucin in the large intestine (Fig. 1F) (Johansson et al. 2009). This protein was erroneously named immunoglobulin G Fc-binding protein. It contains 13 VWD domains that are closely related to the VWD4 domain of MUC2 as 11 of 13 of the VWD contain a GD/PH sequence that can be cleaved like the VWD4 of MUC2. The cleaved FCGBP parts remain associated by disulfide bonds. The amino-terminal portion of the FCGBP was found covalently attached to MUC2, which seems compatible with binding via the emergent carboxy-terminal aspartates. Also in this case, there is no detailed information on how and where FCGBP is bound, but its structure suggests that it can cross-link several MUC2 mucins to stabilize the mucus gel.

To summarize, our model of mature MUC2 mucin suggests a well-organized netlike gel that is cross-linked by five different covalent bonds and one noncovalent bond. It also suggests that the mucus is self-organized, as determined by properties inherent in the MUC2 mucin molecule.

\section{PACKING OF MUCINS}

The oligomeric status of the mucin amino termini is not only important for mucus, but also for how mucins are stored in the goblet cell granules. The von Willebrand factor multimers are packed as long spirals that make up the Weibel-Palade bodies (vesicles) in endothelial cells. The von Willebrand factor is unpacked by a slow unwinding of these spirals into a long polymeric "rope" that is pulled out by and follows the blood flow (Huang et al. 2008; Sadler 2009). This slow unwinding differs from the release of mucins in which mucin granules are released by a quick burst within seconds or milliseconds. To avoid severe mucin entanglement that would hinder the 1000-fold expansion in volume on release, the mucin release must be well organized. Thus mucins must be packed differently from the von Willebrand factor protein.

We recently showed that the MUC2 aminoterminal mucin is packed in goblet cell granules as condensed concatenated rings formed by one amino-terminal trimer in each corner of largely six-sided rings, but five- and seven-sided rings have also been observed (Ambort et al. 2012). The amino-terminal covalent trimers (Fig. 2, blue) interact by noncovalent forces generated after the VWD1 and VWD2 domains are folded back onto the VWD3 domain. This organization in amino-terminal rings is induced by high $\mathrm{Ca}^{2+}$-ion concentration and low $\mathrm{pH}$ created along the secretory pathway. In our experimental system, rings were formed by $50 \mathrm{mM} \mathrm{Ca}{ }^{2+}$ and a pH of 6.2 (Ambort et al. 2012). These rings concatenate into relatively flat surfaces from which the rest of the mucin extends vertically (or perpendicularly) as illustrated in Fig. 2. In this model, the MUC2 mucin is shown in a two-dimensional (2D) reconstruction, and the mucin domains as extended linear rectangles (rods in 3D). The dimeric carboxyl termini of the MUC2 mucins are joined at the other end (Fig. 2A). This model gives the expected, wellorganized packing of mucins in the granules, in contrast to previous models of mucin packing (Verdugo et al. 1987), which was suggested to be solely because of shielding of negative charges. Nevertheless, in our model, mucins with negatively charged $O$ glycans must have

\section{A Packed MUC2 (side)}

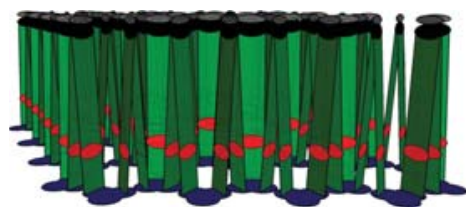

B Packed MUC2 (bottom)

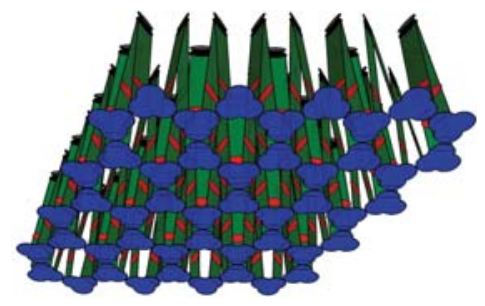

Figure 2. Schematic model of the packed assembled MUC2 polymer as found in the mucin granules of the goblet cells. (A) Side view, and (B) bottom view. The MUC2 mucin is shown in a 2D projection. Colors as in Figure 1. 
D. Ambort et al.

these repulsive electrostatic forces minimized by counterions such as sodium and calcium ions. However, packed mucins cannot be highly organized by solely electrostatic divalent cross-links between mucin glycans, and are better explained by the model in Figure 2.

\section{THE ORGANIZED RELEASE OF MUCINS TO FORM THE MUCUS}

Mucin packing as described in Fig. 2 is compatible with rapid mucin expansion and quick release when the $\mathrm{Ca}^{2+}$ ions are removed and $\mathrm{pH}$ increased. When these cations are removed, the noncovalent interactions maintaining the concatenated ring structure is weakened and the trimeric amino termini start to separate, as illustrated in Fig. 3 (Ambort et al. 2012). When the amino termini start to separate, the dimeric MUC2 carboxy-terminal end "descends" and allows the trimeric amino terminals to spread apart until the net becomes flat. The unfolded mucin will, because of its dimeric carboxy termini and trimeric amino termini, spontaneously form a flat netlike structure with an inherent tendency to spontaneously form sheets (Fig. $4 \mathrm{~A})$. The organization of mucus found in colon is likely to be of this type. Studies using both electron microscopy and fluorescent microsco-

A Unpacking MUC2 (side)
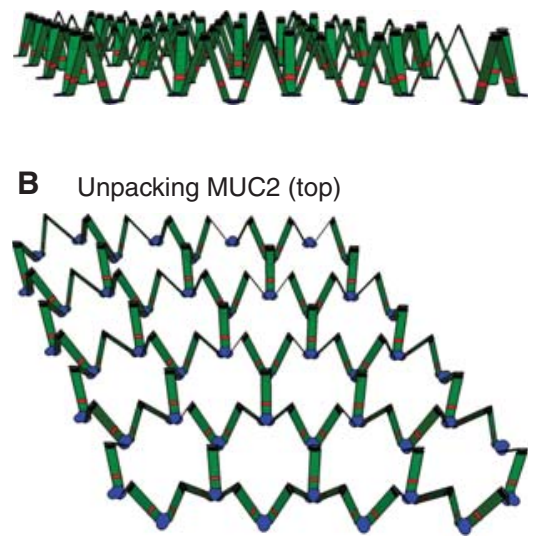

Figure 3. Schematic model of an early stage of unpacking of the assembled MUC2. (A) Side view, and (B) top view. The MUC2 mucin is shown in a $2 \mathrm{D}$ projection. Colors as in Figure 1.
A Unpacked MUC2

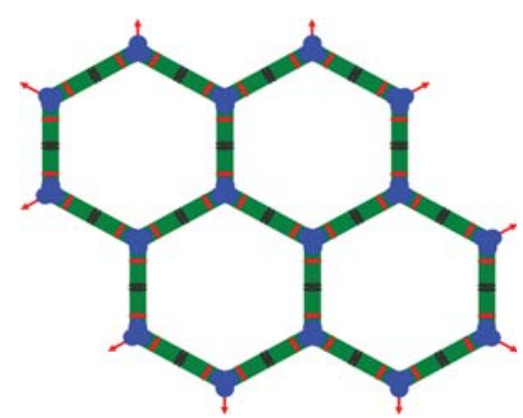

B Stratified MUC2

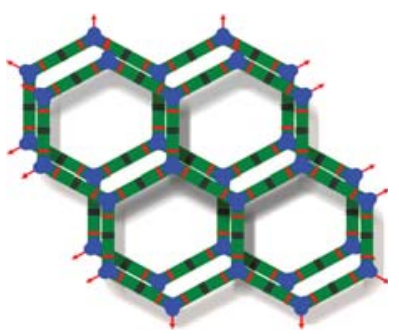

Figure 4. Model of expected organization of the stratified inner mucus layer of colon. Schematic drawing of the fully unfolded secreted MUC2 polymer netlike network $(A)$ formed by the disulfide-bonded trimeric amino termini and dimeric carboxyl termini. $(B)$ The dimerization of the CysD domains will interact maximally if each layer of MUC2 polymer rings is partly dislocated.

py support the case and show that the inner mucus layer is a laminated, stratified structure (Johansson et al. 2008; Ambort et al. 2012). Thus, we propose a model in which the mucins tend to spontaneously, upon secretion, reorganize into flat, netlike structures.

With these concepts in mind, the netlike structure of the MUC2 mucin forms stacked sheets, and with each layer of the net shifted slightly the CysD domains should fit together (Fig. 4B). Although we have no formal proof of this structure, it is tempting to use it to explain the molecular sieving property of the inner mucus layer of the colon. Typically, the CysD domain is located between mucin domains, and thus, the length of the extended mucin domains determines the pore size of the mucus. Interestingly, the MUC2 mucin that is found in intestines that must permeate nutrients have longer 
mucin domains, whereas MUC5AC and 5B mucins found in stomach and lungs, where only gases, water, and ions must permeate, have shorter mucin domains between the CysDs.

The model in Fig. 4 represents our current understanding of how MUC2 mucin is organized in the inner colon mucus layer. However, there is more to be discovered in how the actual mucus gel is organized. Proteolytic cleavage within the mucin polymers is an important additional event that will clearly have a large impact on the mucus structure. These processes will alter the mucus properties. Although we do not have any molecular details yet, it is clear that the conversion from the inner to the outer mucus layer of colon as well as the fourfold expansion in volume found in colon mucus is owing to proteolytic activities (Johansson et al. 2008, 2011). These proteolytic activities do not necessarily lead to polymer disruption as these can cleave the MUC2 in positions in which the intramolecular disulfide bonds can still maintain a covalent polymeric network, although the more penetrable mucus will also allow bacteria to enter (Johansson et al. 2011).

DIOS is a relatively common, poorly understood problem for CF patients. The mucus of the small intestine is, just as for colon, built around the MUC2 mucin. However, small intestinal mucus does not show an inner mucus layer like the colon (Atuma et al. 2001; Gustafsson et al. 2012). Instead, it has only one mucus layer that is penetrable to bacteria (Johansson and Hansson 2011). How MUC2 mucin generates such different mucus properties is still not fully understood, but most likely the differences between the large and small intestine are owing to specific proteolytic processing of MUC2 in the small intestine.

\section{THE IMPORTANCE OF BICARBONATE}

As discussed, the unpacking of mucins requires removal of $\mathrm{Ca}^{2+}$ and an increased $\mathrm{pH}$. The natural agent that can accomplish this is $\mathrm{HCO}_{3}^{-}$. The capacity of cystic fibrosis transmembrane conductance regulator (CFTR) to support $\mathrm{HCO}_{3}^{-}$transport will thus be very important for mucin release as pointed out by Quinton
(2008) and shown for both the cervix and small intestine (Garcia et al. 2009; Muchekehu and Quinton 2010). We have recently shown that the ileal mucus of CF mice appears anchored to the epithelium (Gustafsson et al. 2012), which is in contrast to the small intestine of wild-type animals where the mucus is easily aspirated and penetrable to beads the size of bacteria. In the CF mice, the mucus is two to three times more dense than normal (measured as MUC2 content per volume) and is impermeable to beads the size of bacteria. Remarkably, CF mucus properties appear to be reverted to normal when the mucins are excreted into $115 \mathrm{~mm} \mathrm{HCO}_{3}^{-}$or $20 \mathrm{~mm}$ EDTA (Gustafsson et al. 2012). The volume of the CF mucus also expands more normally when $\mathrm{HCO}_{3}^{-}$is added to luminal fluid, which does not occur with wild-type mucus and indicates that the mucus is only partly expanded.

\section{DISCUSSION}

Although the above discussion takes its start in the biochemical nature and properties of the intestinal MUC2 mucin, there is no doubt that this knowledge can be applied also to the respiratory mucus. The surface mucin of the trachea is MUC5AC, the mucin with the most structural similarities to MUC2. We note that the MUC5AC mucin normally appears to form only one type of mucus in the lungs, whereas it too can form a two-layered system in the stomach, and thereby is similar to the different layers of the MUC2 mucin in the small and large intestine.

This review should be taken together with the views by Verdugo (2012). Our different views of mucus and mucins are obvious, and present in two different models: perfectly linear molecules or complex heavily cross-linked polymeric networks. In other aspects we are in agreement; for example, the importance of $\mathrm{Ca}^{2+}$ chelation for mucin release, although the explanations for the effects differ. Verdugo believes the major effect of $\mathrm{Ca}^{2+}$ is to shield the negative charges of the $O$ glycans, whereas we believe the major effect is on the amino-terminal portion of the mucins as packed in the 
D. Ambort et al.

mucin granules. Despite our expertise in glycan chemistry, we believe that glycans are not that important for mucus formation, which contrasts directly with Verdugo. We do not claim that any of these models give a final answer, but that there are elements in both views that are likely correct. The highly cross-linked mucus is probably converted to less cross-linked forms by well-controlled proteolytic activities and thus mucus will become more linear, although it would never reach an ideal random linear thread. However, many biochemical details are still unknown. We have not yet studied the polymeric organization of any of the other gel-forming mucins or the packing organization in their storage granules. Mucins are complex and it will take quite some time until we are able to present a final and comprehensive model of normal mucus gel or of that found in CF patients. However, the importance of the CFTR channel for transporting bicarbonate for proper mucin release will provide novel therapeutic possibilities also, without a full understanding of the mucus gel.

\section{ACKNOWLEDGMENTS}

This work is supported by the Swedish Research Council (Nos. 7461, 21027), the Swedish Cancer Foundation, the Knut and Alice Wallenberg Foundation, IngaBritt and Arne Lundberg Foundation, Sahlgren's University Hospital (LUA-ALF), Wilhelm and Martina Lundgren's Foundation, Torsten och Ragnar Söderbergs Stiftelser, the Sahlgrenska Academy, National Institute of Allergy and Infectious Diseases (U01AI095473), the Swedish Foundation for Strategic ResearchThe Mucus-Bacteria-Colitis Center (MBC) of the Innate Immunity Program, the Swedish CF Foundation, the U.S. CF Foundation, Erica Lederhausen's Foundation, and Lederhausen's Center for CF Research at the University of Gothenburg.

\section{REFERENCES}

* Reference is also in this collection.

Ambort D, van der Post S, Johansson MEV, MacKenzie J, Thomsson E, Krengel U, Hansson GC. 2011. Function of the CysD domain of the gel-forming MUC2 mucin. Biochem J 436: 61-70.
Ambort D, Johansson MEV, Gustafsson JK, Nilsson H, Ermund A, Johansson BR, Kock P, Hebert H, Hansson GC. 2012. Calcium and pH-dependent packing and release of the gel-forming MUC2 mucin. Proc Natl Acad Sci doi: $10.1073 /$ pnas.1120269109.

Andersch-Björkman Y, Thomsson KA, Holmén Larsson JM, Ekerhovd E, Hansson GC. 2007. Large-scale identification of proteins, mucins and their $O$-glycosylation in the endocervical mucus during the menstrual cycle. Mol Cell Proteomics 6: 708-716.

Asker N, Baeckstrom D, Axelsson MAB, Carlstedt I, Hansson GC. 1995. The human MUC2 mucin apoprotein appears to dimerize before O-glycosylation and shares epitopes with the "insoluble" mucin of rat small intestine. Biochem J 308: 873-880.

Asker N, Axelsson MAB, Olofsson SO, Hansson GC. 1998. Human MUC5AC mucin dimerizes in the rough endoplasmic reticulum, similarly to the MUC2 mucin. Biochem J 335: 381-387.

Atuma C, Strugula V, Allen A, Holm L. 2001. The adherent gastrointestinal mucus gel layer: Thickness and physical state in vivo. Am J Physiol 280: G922-G929.

Axelsson MAB, Asker N, Hansson GC. 1998. O-glycosylated MUC2 monomer and dimer from LS 174T cells are water-soluble, whereas larger MUC2 species formed early during biosynthesis are insoluble and contain nonreducible intermolecular bonds. J Biol Chem 273: 1886418870 .

Carlstedt I, Herrmann A, Karlsson H, Sheehan JK, Fransson L, Hansson GC. 1993. Characterization of two different glycosylated domains from the insoluble mucin complex of rat small intestine. J Biol Chem 268: 1877118781 .

Gagneux P, Varki A. 1999. Evolutionary considerations in relating oligosaccharide diversity to biological function. Glycobiology 9: 747-755.

Garcia MA, Yang N, Quinton PM. 2009. Normal mouse intestinal mucus release requires cystic fibrosis transmembrane regulator-dependent bicarbonate secretion. J Clin Invest 119: 2613-2622.

Godl K, Johansson MEV, Karlsson H, Morgelin M, Lidell ME, Olson FJ, Gum JR, Kim YS, Hansson GC. 2002. The N-termini of the MUC2 mucin form trimers that are held together within a trypsin-resistant core fragment. J Biol Chem 277: 47248-47256.

Gum JR, Hicks JW, Toribara NW, Siddiki B, Kim YS. 1994. Molecular cloning of human intestinal mucin (MUC2) cDNA. Identification of the amino terminus and overall sequence similarity to prepro-von Willebrand factor. J Biol Chem 269: 2440-2446.

Gustafsson JK, Ermund A, Ambort D, Johansson MEV, Nilsson HE, Thorell K, Hebert H, Sjovall H, Hansson GC. 2012. Bicarbonate and functional CFTR channel are required for proper mucin secretion and link Cystic Fibrosis with its mucus phenotype. J Exp Med (in press).

Heazlewood CK, Cook MC, Eri R, Price GR, Tauro SB, Taupin D, Thornton DJ, Png CW, Crockford TL, Cornall RJ, et al. 2008. Aberrant mucin assembly in mice causes endoplasmic reticulum stress and spontaneous inflammation resembling ulcerative colitis. PLoS Med 5: e54.

Herrmann A, Davies JR, Lindell G, Martensson S, Packer NH, Swallow DM, Carlstedt I. 1999. Studies on the 
"insoluble" glycoprotein complex from human colon. J Biol Chem 274: 15828-15836.

Holmen Larsson JM, Karlsson H, Sjovall H, Hansson GC. 2009. A complex, but uniform $O$-glycosylation of the human MUC2 mucin from colonic biopsies analyzed by nanoLC/MS ${ }^{\mathrm{n}}$. Glycobiology 19: 756-766.

Huang RH, Wang Y, Roth R, Yu X, Purvis AR, Heuser JE, Egelman EH, Sadler JE. 2008. Assembly of Weibel Palade body-like tubules from N-terminal domains of von Willebrand factor. Proc Natl Acad Sci 105: 482-487.

Johansson MEV, Hansson GC. 2011. Keeping bacteria at a distance. Science 334: 182-183.

Johansson MEV, Phillipson M, Petersson J, Holm L, Velcich A, Hansson GC. 2008. The inner of the two Muc2 mucin dependent mucus layers in colon is devoid of bacteria. Proc Natl Acad Sci 105: 15064-15069.

Johansson MEV, Thomsson KA, Hansson GC. 2009. Proteomic analyses of the two mucus layers of the colon barrier reveal that their main component, the Muc2 mucin, is strongly bound to the Fcgbp protein. J Proteome Res 8: 3549-3557.

Johansson MEV, Holmen Larsson JM, Hansson GC. 2011. The two mucus layers of colon are organized by the MUC2 mucin, whereas the outer layer is a legislator of host-microbial interactions. Proc Natl Acad Sci 108: 4659-4665.

* Kreda SM, Davis CW, Rose MC. 2012. CFTR, mucins, and mucus obstruction in cystic fibrosis. Cold Spring Harb Perspect Med doi: 10.1101/cshperspect.a009589.

Lang T, Alexandersson M, Hansson GC, Samuelsson T. 2004. Bioinformatic identification of polymerizing and transmembrane mucins in the puffer fish Fugu rubripes. Glycobiology 14: 521-527.

Lang T, Hansson GC, Samuelsson T. 2007. Gel-forming mucins appeared early in metazoan evolution. Proc Natl Acad Sci 104: 16209-16214.

Leir SH, Harris A. 2011. MUC6 mucin expression inhibits tumor cell invasion. Exp Cell Res 317: 2408-2419.

Lidell ME, Hansson GC. 2006. Cleavage in the GDPH sequence of the C-terminal cysteine-rich part of the human MUC5AC mucin. Biochem J 399: 121-129.
Lidell ME, Johansson MEV, Hansson GC. 2003a. An autocatalytic cleavage in the C-terminus of the human MUC2 mucin occurs at the low $\mathrm{pH}$ of the late secretory pathway. J Biol Chem 278: 13944-13951.

Lidell ME, Johansson MEV, Mörgelin M, Asker N, Gum JR, Kim YS, Hansson GC. 2003b. The recombinant C-terminus of the human MUC2 mucin forms dimers in $\mathrm{CHO}$ cells and heterodimers with full-length MUC2 in LS 174T cells. Biochem J 372: 335-345.

Muchekehu RW, Quinton PM. 2010. A new role for bicarbonate secretion in cervico-uterine mucus release. J Physiol 588: 2329-2342.

Perez-Vilar J, Hill RL. 1998. Identification of the half-cystine residues in porchine submaxillary mucin critical for multimerization through the D-domains. J Biol Chem 273: 34527-34534.

Perez-Vilar J, Randell SH, Boucher RC. 2004. C-mannosylation of MUC5AC and MUC5B Cys subdomains. Glycobiology 14: 325-337.

Quinton PM. 2008. Cystic fibrosis: Impaired bicarbonate secretion and mucoviscidosis. Lancet 372: 415-417.

Sadler JE. 2009. von Willebrand factor assembly and secretion. J Thromb Haemost 7: 24-27.

Sheehan JK, Kirkham S, Howard M, Woodman P, Kutay S, Brazeau C, Buckley J, Thornton DJ. 2004. Identification of molecular intermediates in the assembly pathway of the MUC5AC mucin. J Biol Chem 279: $15698-$ 15705.

Thornton DJ, Rousseau K, McGuckin MG. 2008. Structure and function of the polymeric mucins in airways mucus. Ann Rev Physiol 70: 459-486.

* Verdugo P. 2012. Supramolecular dynamics of mucus. Cold Spring Harb Perspect Med doi: 10.1101/cshperspect. a009597.

Verdugo P, Deyrup-Olsen I, Aitken M, Villalon M, Johnson D. 1987. Molecular mechanism of mucin secretion: 1. The role of intragranular charge shielding. J Dental Res 66: 506-508. 


\title{
$\&_{\mathrm{CSH}}^{\infty} \&$ Cold Spring Harbor

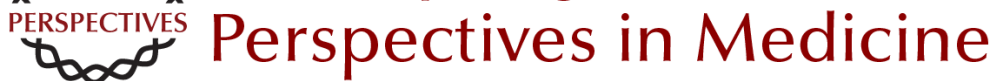

\section{Perspectives on Mucus Properties and Formation--Lessons from the Biochemical World}

\author{
Daniel Ambort, Malin E.V. Johansson, Jenny K. Gustafsson, Anna Ermund and Gunnar C. Hansson
}

Cold Spring Harb Perspect Med 2012; doi: 10.1101/cshperspect.a014159

Subject Collection Cystic Fibrosis

Antibiotic and Anti-Inflammatory Therapies for

Cystic Fibrosis

James F. Chmiel, Michael W. Konstan and J. Stuart Elborn

Structure and Function of the Mucus Clearance

System of the Lung

Brenda M. Button and Brian Button

New Pulmonary Therapies Directed at Targets

Other than CFTR

Scott H. Donaldson and Luis Galietta

The Cystic Fibrosis Airway Microbiome

Susan V. Lynch and Kenneth D. Bruce

Cystic Fibrosis Transmembrane Conductance Regulator (ABCC7) Structure John F. Hunt, Chi Wang and Robert C. Ford

Status of Fluid and Electrolyte Absorption in Cystic Fibrosis

M.M. Reddy and M. Jackson Stutts

The Influence of Genetics on Cystic Fibrosis

Phenotypes

Michael R. Knowles and Mitchell Drumm

Perspectives on Mucus Properties and Formation

--Lessons from the Biochemical World Daniel Ambort, Malin E.V. Johansson, Jenny K.

Gustafsson, et al.
The Cystic Fibrosis Intestine

Robert C. De Lisle and Drucy Borowitz

Cystic Fibrosis Transmembrane Regulator

Correctors and Potentiators

Steven M. Rowe and Alan S. Verkman

The Cystic Fibrosis of Exocrine Pancreas

Michael Wilschanski and Ivana Novak

Dynamics Intrinsic to Cystic Fibrosis

Transmembrane Conductance Regulator Function and Stability

$P$. Andrew Chong, Pradeep Kota, Nikolay V.

Dokholyan, et al.

The Cystic Fibrosis Gene: A Molecular Genetic

Perspective

Lap-Chee Tsui and Ruslan Dorfman

The CFTR Ion Channel: Gating, Regulation, and

Anion Permeation

Tzyh-Chang Hwang and Kevin L. Kirk

Assessing the Disease-Liability of Mutations in

CFTR

Claude Ferec and Garry R. Cutting

Supramolecular Dynamics of Mucus

Pedro Verdugo

For additional articles in this collection, see http://perspectivesinmedicine.cshlp.org/cgi/collection/ 